\title{
The effects of regular visual contact with human beings on fear, stress, antibody and growth responses in broiler chickens
}

\begin{abstract}
This study investigated the effects of regular visual contact at various ages on tonic immobility (TI) and heterophil/lymphocyte ratio (HLR) reactions to handling and crating, antibody production, growth performance, and feed conversion ratios in commercial broiler chicks. Broiler chicks were exposed to (1) visual contact with an experimenter for $10 \mathrm{~min}$ twice daily from 0 to 3 weeks (V0ï 3), (2) visual contact with an experimenter for 10 min twice daily from 0 to 6 weeks (V0ï 6), (3) visual contact with an experimenter for 10 min twice daily from 3 to 6 weeks (V3ï 6) and (4) no visual contact (NV), as control. At 42 days of age, the birds were captured and crated for $10 \mathrm{~min}$. Following, the $10 \mathrm{~min}$ crating, the V0Ï 3 and VOÏ 6 birds had shorter TI duration and lower HLR than those of NV. Although, the HLR response of V3ï 6 birds to crating were similar to their V0Ï 3 and V0Ï 6 counterparts, the latter two groups were less fearful, as measured by TI duration. Subjecting birds to V0ï 3 and V0Ï 6 enhanced antibody response to Newcastle disease vaccine. Regular visual contact had negligible influence on body weight, feed conversion ratios and survivability of birds. Collectively, the results suggest that regular visual contact from 1 to 21 days of age is sufficient to reduce fear and stress reactions to handling and crating, and improve antibody response in broiler chickens.
\end{abstract}

Keyword: Broiler chickens; Visual contact; Fear; Stress; Performance; Immunity 